\title{
The role of a ginseng saponin metabolite as a DNA methyltransferase inhibitor in colorectal cancer cells
}

\author{
KYOUNG AH KANG ${ }^{1}$, HEE SUN KIM ${ }^{2}$, DONG HYUN KIM ${ }^{3}$ and JIN WON HYUN ${ }^{1}$ \\ ${ }^{1}$ School of Medicine and Institute for Nuclear Science and Technology, Jeju National University, Jeju 690-756; \\ ${ }^{2}$ Department of Neuroscience, College of Medicine, Ewha Womans University, Seoul 110-783; ${ }^{3}$ Department of \\ Microbial Chemistry, College of Pharmacy, Kyung Hee University, Seoul 130-701, Republic of Korea
}

Received January 17, 2013; Accepted March 15, 2013

DOI: 10.3892/ijo.2013.1931

\begin{abstract}
Hypermethylation of runt-related transcription factor 3 (RUNX3) promoter regions occurs in at least $65 \%$ of colorectal cancer cell lines. Compound K, the main metabolite of ginseng saponin, induced demethylation of a RUNX3 promoter in HT-29 human colorectal cancer cells, assessed by methylation-specific PCR and the quantitative pyrosequencing analysis. The demethylation of RUNX3 in compound K-treated cells resulted in the re-expression of RUNX3 mRNA, protein and the localization into the nucleus. Demethylation of the RUNX3 gene by compound K occurred via inhibition of the expression and activity of DNA methyltransferase 1 (DNMT1). Compound $\mathrm{K}$ also significantly induced RUNX3-mediated expression of Smad4 and Bim. DNMT1 inhibitory activity by compound $\mathrm{K}$ was related to extracellular signal-regulated kinase (ERK) inhibition, assessed by siRNA transfection on DNMT1 and ERK. In conclusion, compound K significantly inhibits the growth of colorectal cancer cells by inhibiting DNMT1 and reactivating epigenetically-silenced genes. Ginseng saponin is a potential candidate as DNMT1 inhibitor in the chemoprevention of cancer.
\end{abstract}

\section{Introduction}

Colorectal carcinoma (CRC) is one of the most common and well-studied malignancies in the Western world. The disease is thought to originate in multi-potential stem cells located in the intestinal crypts; (non-) polypoid precursor lesions initiate from these cells, and metastatic CRC can develop $(1,2)$. The histological progression of colorectal carcinogenesis is characterized by sequential genetic (3) and epigenetic alterations $(4,5)$. To date, intensive efforts have been made by scientists to find a provocative factor of this major cancer; many epidemiologic

Correspondence to: Professor Jin Won Hyun, School of Medicine, Jeju National University, Jeju 690-756, Republic of Korea E-mail: jinwonh@jejunu.ac.kr

Key words: DNA methyltransferase, ginseng saponin metabolite, colorectal cancer, tumor suppressor gene, epigenetic alteration studies indicate that a Western-style diet is associated with a high incidence of CRC (6,7).

The development of CRC is usually described as a multistep model, in which the accumulation of genetic and epigenetic events mediates the adenoma-carcinoma sequence (8). The accumulation of mutations is driven through distinct pathways by different types of genomic instability, chromosomal instability and microsatellite instability (9). Additionally, an epigenetic pathway has been proposed, in which tumor suppressor genes are inactivated by promoter methylation and silencing of gene transcription (10).

Runt-related transcription factor 3 (RUNX3) belongs to the RUNX family of genes, which is important in mammalian development and neoplasia (11-13). RUNX3 cooperates with Sma and Mad related family $3(\operatorname{Smad} 3) / \operatorname{Smad} 4$ to activate transforming growth factor- $\beta$ (TGF- $\beta$ )-dependent growth inhibition and apoptosis by inducing p21 and Bim (14). The RUNX3 gene is localized to the $1 \mathrm{p} 36$ locus and is linked with gastric epithelial homeostasis and gastric carcinogenesis. The $1 \mathrm{p} 36$ region is thought to harbor at least one tumor suppressor gene, since this region exhibits frequent loss of heterozygosity in colon, gastric, breast and ovarian cancers (15). Additionally, the introduction of a normal human $1 \mathrm{p} 36$ chromosome fragment into colon cancer cells suppresses their tumorigenicity (16). Interestingly, a considerable proportion of gastric cancers do not express RUNX3 due to hemizygous deletion and hypermethylation of the RUNX3 promoter region (17). Hypermethylation of the RUNX3 promoter occurs in $21 \%$ of colon cancer specimens and at least $65 \%$ of colon cancer cell lines, suggesting that RUNX3 has a tumor suppressive function in CRC (18).

The regulation of DNA methylation is not well understood, but involves DNA methyltransferases (DNMTs), which catalyze the transfer of methyl groups to the carbon-5 position of cytosines in phosphodiester bond between the cytosine and the guanine $(\mathrm{CpG})$ islands. Three active DNMTs have been identified in mammals, DNMT1, DNMT3A and DNMT3B. DNMT1 is largely responsible for maintaining methylation, and contributes to de novo DNA promoter methylation in cancer (19). Some models suggest that DNMT3B cooperates with DNMT1 to maintain DNA methylation status (20). Epigenetic changes by DNMTs are highly relevant to colon carcinogenesis; as such, they represent a target for novel strate- 
gies that can prevent or treat cancer. DNA methylation results in the local recruitment of histone deacetylases to promoter regions, with co-localization of methyl CpG binding protein 2 . These eventually inhibit the binding of RNA polymerase II, thereby repressing the expression of genes and their products. Such observations led to the paradigm that DNA methylation occurs upstream of histone acetylation in this pathway of transcriptional repression. DNMTs produce methylation of $\mathrm{CpG}$ repeats in the upstream promoter regions of genes, while physiological upstream regulators of the activity and levels of DNMTs have not yet been identified.

Recent studies suggest that DNA methylation in colon cancer and NIH 3 T3 cells may be regulated, in some circumstances by extracellular signal-regulated kinase (ERK) activity (21). DNA methylation and DNMTs might be modulated directly or indirectly by the extracellular milieu through signal transduction pathways, which may include ERK or other cellular kinases.

Recently, two well-characterized and clinically-relevant DNMT inhibitors, 5-aza-cytidine (5-Aza) and its metabolite 5-aza-2'deoxycytidine (5-Aza-2'dC), were identified as nucleoside analogue mechanism-based inhibitors. They are substituted for cytosine residues in the DNA strand during replication, and inhibit DNMT activity by covalently binding to the DNMT enzymes, resulting in reduced genomic DNA methylation (22,23). The Food and Drug Administration has approved these two inhibitors for the treatment of myelodysplastic syndromes (24). Although effective against certain hematopoietic disorders, these drugs show some toxicity both in vitro and in vivo, and are unstable in neutral solutions (25-27). The inherent lack of specificity of 5-Aza and 5-Aza-2' $\mathrm{dC}$ for their target genes allows for these undesirable effects. For example, global demethylation by 5-Aza and 5-Aza-2'dC may result in the expression of oncogenic loci and activation of transposable elements, inducing the expression of multidrug resistance genes (28-30). Hence, a non-toxic, highly stable, and effective DNMT inhibitor would be an ideal epigenetic therapeutic agent.

A number of saponins have been isolated from ginseng and extensively investigated with regard to their possible antitumor activity in various cancer cell lines (31-33). Among them, 20-O-( $\beta$-D-glucopyranosyl)-20(S)-protopanaxadiol (compound K, Fig. 1) is the main metabolite of protopanaxadiol-type ginsenoside, formed in the intestine after oral administration (34-36). We recently reported that compound $\mathrm{K}$ exhibited cytotoxicity by inducing apoptosis, arrest of growth at the G1 phase of the cell cycle, and inhibition of telomerase activity in human leukemia cells $(37,38)$. In addition, combined treatment with compound $\mathrm{K}$ and $\gamma$-irradiation enhanced cell death in human lung cancer cells (39). Finally, compound K induced apoptosis in MCF-7 breast cancer cells by modulating AMP-activated protein kinase (40).

The aim of this study was to determine whether compound $\mathrm{K}$ reactivates tumor suppressor genes in human colorectal cancer HT-29 cells by inhibiting DNMT. The results demonstrate that compound $\mathrm{K}$ treatment results in demethylation of the silenced gene, RUNX3, via inhibition of ERK and recruitment of RUNX3-mediated proteins, such as Smad4 and Bim. These results show, for the first time, that compound $\mathrm{K}$ alters DNA methylation patterns within the RUNX3 gene promoter.

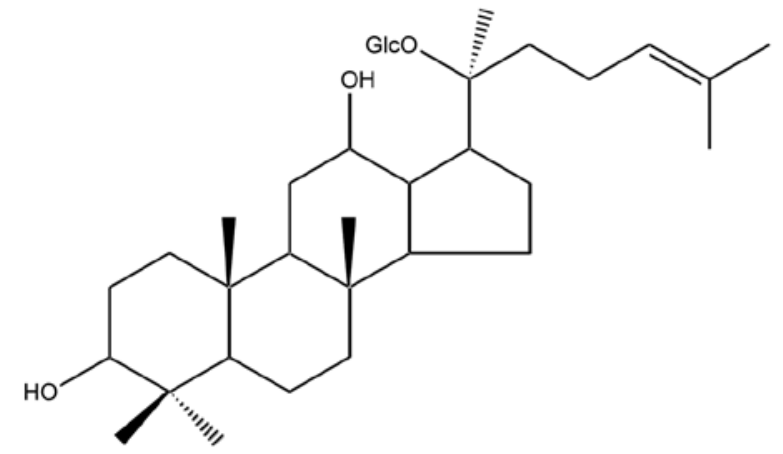

Figure 1. Chemical structure of Compound K [20-O-D-glucopyranosyl-20(S)protopanaxadiol].

\section{Materials and methods}

Cell culture. Human colorectal cancer HT-29 cells were obtained from the Korean Cell Line Bank (Seoul, Republic of Korea). Cells were maintained in an incubator at $37^{\circ} \mathrm{C}$ with a humidified atmosphere of $5 \% \mathrm{CO}_{2}$. The cells were cultured in RPMI-1640 medium containing 10\% fetal calf serum, streptomycin $(100 \mu \mathrm{g} / \mathrm{ml})$ and penicillin $(100 \mathrm{U} / \mathrm{ml})$.

Cell proliferation assay. The effect of compound $\mathrm{K}$ on the proliferation of the cells was determined using the 3-(4,5-dimethylthiazol-2-yl)-2,5-diphenyltetrazolium bromide (MTT) assay. Cells were seeded in a 96-well plate at a density of $1 \times 10^{5}$ cells $/ \mathrm{ml}$ and treated with compound $\mathrm{K}$. After incubating for $48 \mathrm{~h}, 50 \mu \mathrm{l}$ of the MTT stock solution $(2 \mathrm{mg} / \mathrm{ml})$ was added to each well to attain a total reaction volume of $250 \mu \mathrm{l}$. After $4 \mathrm{~h}$ incubation, the supernatants were aspirated. The formazan crystals in each well were then dissolved in $150 \mu \mathrm{l}$ dimethylsulfoxide and absorbance at $540 \mathrm{~nm}$ was read on a scanning multi-well spectrophotometer.

Reverse transcription polymerase chain reaction (RT-PCR). Total RNA was isolated from cells using TRIzol reagent (Gibco-BRL, Grand Island, NY, USA). Complementary DNA (cDNA $1 \mu \mathrm{l}$ ) was amplified in a reverse-transcription reaction containing primers, dNTPs, and 0.5 units of Taq DNA polymerase at a final volume of $25 \mu \mathrm{l}$. The PCR conditions were: $5 \mathrm{~min}$ at $94^{\circ} \mathrm{C}$ for initial denaturation, followed by 35 cycles of $1 \mathrm{~min}$ at $94^{\circ} \mathrm{C}, 1 \mathrm{~min}$ at $55^{\circ} \mathrm{C}$ and $1 \mathrm{~min}$ at $72^{\circ} \mathrm{C}$, and a final elongation period of $7 \mathrm{~min}$ at $72^{\circ} \mathrm{C}$. PCR amplification was carried out in a programmable thermal cycler (Perkin-Elmer Cetus 9600, Roche Molecular Systems Inc., Branchburg, NJ, USA). The primers used to amplify the RUNX3 and DNMT1 cDNA $(30,42)$ were: RUNX3 sense, 5'-GGCAATGACGAGAA CTAC-3' (located in exon 2), antisense, 5'-GGAGAATGGGT TCAGTTC-3' (located in exon 5); DNMT1 sense, 5'-CGCTGT ATCTAGCAAGGGTCA-3', antisense, 5'-TCGAATCTCGCG TAGTCTTG-3'; GAPDH sense, 5'-GTGGGCCGCCCTAGG CACCAGG-3', antisense 5'-GGAGGAAGAGGATGCGGC AGTG-3'. The amplified products were resolved on $1 \%$ agarose gels, stained with ethidium bromide and photographed under ultraviolet light, using Image Quant ${ }^{\mathrm{TM}}$ TL analysis software (Amersham Bioscience, Uppsala, Sweden). 
Western blot analysis. Cells were harvested and lysed on ice in $1 \mathrm{ml}$ of lysis buffer $(10 \mathrm{mM}$ Tris- $\mathrm{HCl}, \mathrm{pH} 7.9,10 \mathrm{mM} \mathrm{NaCl}$, $3 \mathrm{mM} \mathrm{MgCl}{ }_{2}$ and $1 \% \mathrm{NP}-40$ ) for $4 \mathrm{~min}$. After centrifugation for $10 \mathrm{~min}$ at $3,000 \mathrm{xg}$, the pellets were re-suspended in $50 \mu \mathrm{l}$ of extraction buffer (20 mM HEPES, pH 7.9, 20\% glycerol, $1.5 \mathrm{mM} \mathrm{MgCl} \mathrm{Mg}_{2}, 0.2 \mathrm{mM}$ EDTA, $1 \mathrm{mM}$ DTT and $1 \mathrm{mM}$ PMSF). This was incubated on ice for $30 \mathrm{~min}$ and centrifuged at $13,000 \mathrm{x} \mathrm{g}$ for $5 \mathrm{~min}$. Following measurement of the protein concentration, supernatants were stored at $-70^{\circ} \mathrm{C}$. Aliquots of the lysates (40 $\mu \mathrm{g}$ of protein) were boiled for $5 \mathrm{~min}$ and electrophoresed on a $10 \%$ SDS-polyacrylamide gel. Proteins were transferred onto nitrocellulose membranes, which were subsequently incubated with primary antibodies. The membranes were further incubated with secondary immunoglobulin-Ghorseradish peroxidase conjugates (Pierce, Rockford, IL, USA). Protein bands were detected using an enhanced chemiluminescence western blot analysis detection kit (Amersham, Little Chalfont, UK). The protein bands were visualized using a luminescent image analyzer.

Methylation-specific (MS)-PCR. The bisulfite modification of DNA was conducted using a Methylamp ${ }^{\text {TM }}$ DNA modification kit (Epigentek, Pittsburgh, PA, USA) according to the manufacturer's instructions. For analysis of DNA methylation of RUNX3, MS-PCR was conducted using an Epitect MSP kit (Qiagen, Valencia, CA, USA). PCR products were separated on $6 \%$ non-denaturing polyacrylamide gels, stained with ethidium bromide, and visualized under UV light. The methylated or unmethylated RUNX3 primer sets were (17): unmethylated RUNX3 sense, 5'-TTATGAGGGGTGGTTG TATGTGGG-3', antisense, 5'-AAAACAACCAACACAAAC ACCTCC-3'; methylated RUNX3 sense, 5'-TTACGAGGGG CGGTCGTACGCGGG-3', antisense, 5'-AAAACGACCGAC GCGAACGCCTCC-3'.

Immunocytochemistry. Cells plated on coverslips were fixed with $4 \%$ paraformaldehyde for $30 \mathrm{~min}$ and permeabilized with $0.1 \%$ Triton X-100 in PBS for 2.5 min. Cells were treated with blocking medium (3\% bovine serum albumin in PBS) for $1 \mathrm{~h}$ and incubated for $2 \mathrm{~h}$ with the RUNX3 antibody diluted in blocking medium. The primary RUNX3 antibody was detected by a FITC-conjugated secondary antibody (1:500; Santa Cruz Biotechnology, Santa Cruz, CA, USA) for $1 \mathrm{~h}$. After washing with PBS, stained cells were mounted onto microscope slides in mounting medium with DAPI (Vector, Burlingame, CA, USA), and imaged using the LSM 510 program on a Zeiss confocal microscope.

DNMT activity. Nuclear extracts were prepared using a nuclear protein extraction kit (Cayman Chemical, Ann Arbor, MI, USA). After measuring the protein concentration, the nuclear fractions were stored at $-70^{\circ} \mathrm{C}$. DNMT activity was detected using a EpiQuik DNA methyltransferase activity assay kit (Epigentek). In this assay, the cytosine-rich DNA substrate is stably coated on the strip wells. DNMT transfers a methyl group from $\mathrm{S}$-adenosylmethionine to a cytosine in the DNA substrate. Methylated DNA is recognized using a 5-methylcytosine antibody. The levels of methylated DNA, which are proportional to enzymatic activity, are then quantified colorimetrically using an ELISA-like reaction. The results are expressed as absorbance units at $450 \mathrm{~nm}$ and represent percentage activity.

Immunoprecipitation. Smad4 was immunoprecipitated from the nuclear extracts using the Smad4 antibody. Immune complexes were collected with protein $\mathrm{G}$ beads and washed with immunoprecipitation buffer. Equal amounts of the precipitates were run on an SDS-polyacrylamide gel followed by western blot analysis with antibodies specific for RUNX3.

Chromatin immunoprecipitation (ChIP) assay. The ChIP assay was performed using a Simple ChIP ${ }^{\text {TM }}$ enzymatic chromatin IP kit (Cell Signaling Technology, Danvers, MA, USA) according to the manufacturer's protocol with slight modifications. Briefly, cells were treated with $20 \mu \mathrm{g} / \mathrm{ml}$ of compound $\mathrm{K}$ for $48 \mathrm{~h}$ and then cross-linked by addition of $1 \%$ formaldehyde. Chromatin was prepared and digested with nuclease for $12 \mathrm{~min}$ at $37^{\circ} \mathrm{C}$. ChIP was performed with the RUNX3 antibody (Abcam, Cambridge, MA, USA) and normal mouse IgG. Antibodies were added to the chromatin digests and incubated with constant rotation overnight at $4^{\circ} \mathrm{C}$. ChIP-grade protein $\mathrm{G}$ magnetic beads were then added to capture the immune complexes. The beads were washed and the immunoprecipitates eluted with ChIP elution buffer. The cross-links were reversed by incubation at $65^{\circ} \mathrm{C}$ for $30 \mathrm{~min}$. Proteinase $\mathrm{K}$ was added and incubated at $65^{\circ} \mathrm{C}$ for $2 \mathrm{~h}$. The immunoprecipitated DNA fragments were then purified using spin columns. DNA recovered from the immuneprecipitated complex was subjected to 35 cycles of PCR. The primers for the Bim (RUNX3 binding site) gene promoter were: sense, 5'-GGCAATGACGAGAACTAC-3'; antisense, 5'-GGAG AATGGGTTCAGTTC-3'. The PCR products were separated on $2 \%$ agarose gels, and DNA bands visualized using the Image program (NIH, Bethesda, MD, USA).

Pyrosequencing analysis. The promoter regions of all genes were amplified using a (biotinylated) forward primer and a (biotinylated) reverse primer, and a sequencing primer designed by PSQ Assay Design (Qiagen); a (biotinylated) sense, 5'-(biotinylated) GGYGGGAGGYGGYGGTAGYGGTATAGTT-3' (Y; T or C); a (biotinylated) antisense, 5'-(biotinylated) RCAACCTACCCR ACTAATCCC-3' (R; A or G); sequencing primer, 5'-RCAACC TACCCR ACTAAT-3'. In addition, genomic DNA (30 ng) was modified by sodium bisulfite using the EZ DNA methylation kit (Zymo Research Co., Irvine, CA, USA) according to the manufacturer's instructions. Bisulfite-modified DNA was amplified in a $50 \mu \mathrm{l}$ reaction using biotinylated primer sets and 5 units of Taq polymerase (Enzynomics Co., Daejeon, Republic of Korea). The samples were heated at $94^{\circ} \mathrm{C}$ for $5 \mathrm{~min}$ and then amplified for 45 cycles consisting of $94^{\circ} \mathrm{C}$ for $30 \mathrm{sec}, 55^{\circ} \mathrm{C}$ for $30 \mathrm{sec}$ and $72^{\circ} \mathrm{C}$ for $30 \mathrm{sec}$. All reactions were then incubated at $72^{\circ} \mathrm{C}$ for $5 \mathrm{~min}$ and cooled to $4^{\circ} \mathrm{C}$. The PCR products were visualized on a $2 \%$ agarose gel by ethidium bromide staining. Pyrosequencing reactions were conducted with sequencing primers on the PyroMark ID (Qiagen) according to the manufacturer's specifications. Unmethylated Cytosine (C) is measured according to the relative $\mathrm{T}$ content at the $\mathrm{CpG}$ site, and methylated Cytosine $(\mathrm{mC})$ is measured according to the relative $\mathrm{C}$ content at the $\mathrm{CpG}$ site. The methylation index of each gene promoter and of each sample was calculated as the average value of $\mathrm{mC} /(\mathrm{mC}+\mathrm{C})$ for all examined CpGs in the target region. 
A

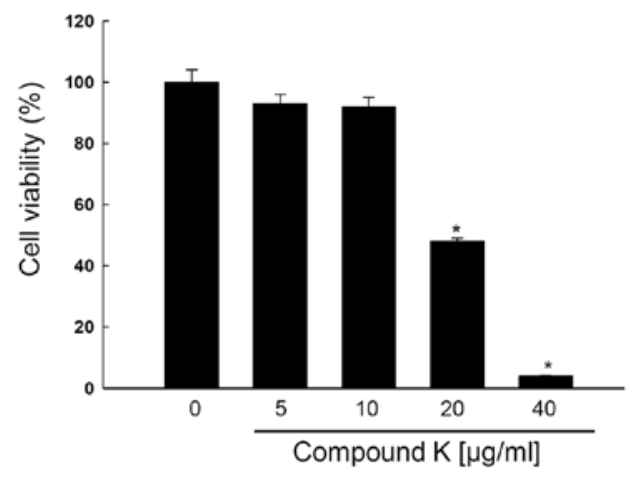

C

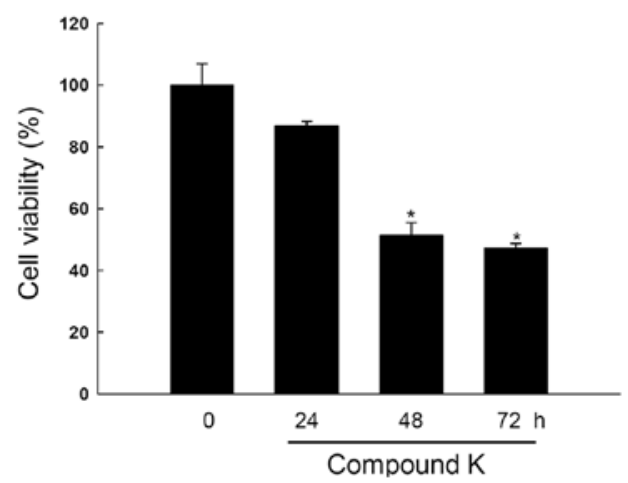

B

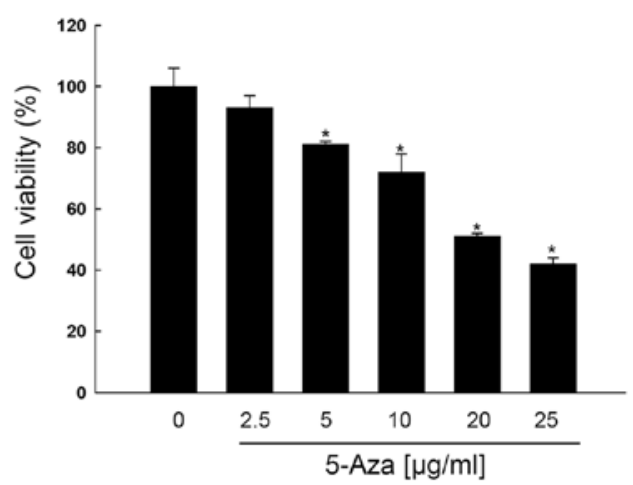

D

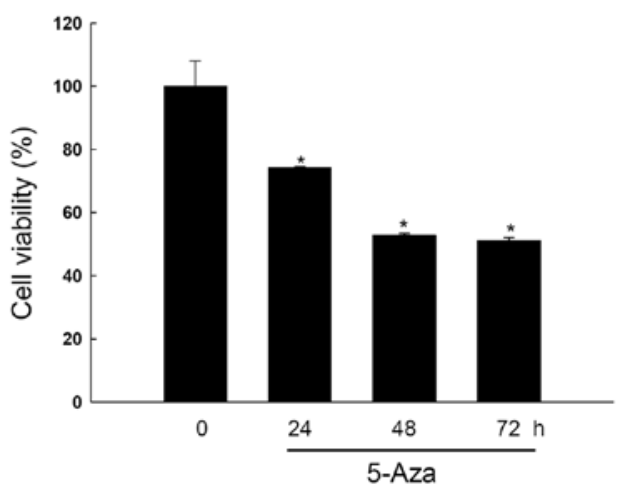

Figure 2. Growth inhibition effects of compound K and 5-Aza in human colon cancer HT-29 cells. Cell viability was assessed at various concentrations of (A) compound $\mathrm{K}$ and (B) 5-Aza at $48 \mathrm{~h}$, and at various times in (C) compound $\mathrm{K}$ at $20 \mu \mathrm{g} / \mathrm{ml}$ and (D) 5-Aza at $19 \mu \mathrm{g} / \mathrm{ml}$ treated cells. Statistical significance was assessed using MTT test. " p< $<.05$, significantly different from the control.

Transient transfection of small RNA interference (siRNA). Cells were seeded at $1.5 \times 10^{5}$ cells/well in 24 -well plates and allowed to reach approximately $50 \%$ confluence on the day of transfection. The siRNA constructs used were: a mismatched siRNA control (siControl; Santa Cruz Biotechnology); a siRNA against ERK, and an siRNA against DNMT1 (Santa Cruz Biotechnology). Cells were transfected with 10-50 nM siRNA using lipofectamine RNAiMax (Invitrogen, Carlsbad, CA, USA) based on the manufacturer's instructions. At $24 \mathrm{~h}$ after transfection, the cells were treated with compound $\mathrm{K}$ for $48 \mathrm{~h}$ and examined by either western blot analysis or MTT assay.

Statistical analysis. All measurements were performed in triplicate and values expressed as the mean \pm the standard error of the mean (SEM). The results were examined using analysis of variance (ANOVA) and Tukey's test to determine pairwise differences. $\mathrm{p}<0.05$ was considered significant.

\section{Results}

Compound $K$ inhibits HT-29 cell growth in a dose- and time-dependent manner. RUNX3 in HT-29 cells is epigenetically silenced (41-45) and the present study used HT-29 cells to elucidate the epigenetic mechanisms of RUNX3. As shown in Fig. 2A and B, compound $\mathrm{K}$ and 5-Aza inhibited
HT-29 cell growth in a dose-dependent manner at 10, 20, 30, $40 \mu \mathrm{g} / \mathrm{ml}$ and $2.5,5,10,20,25 \mu \mathrm{g} / \mathrm{ml}$, respectively, and the concentration that yielded $50 \%$ growth inhibition $\left(\mathrm{IC}_{50}\right)$ was for compound $\mathrm{K} 20 \pm 1.0 \mu \mathrm{g} / \mathrm{ml}$, and for 5 -Aza $19 \pm 0.6 \mu \mathrm{g} / \mathrm{ml}$, and both inhibited cell growth in a time-dependent manner (Fig. 2C and D).

Compound $K$ reverses methylation and reactivates the tumor suppressor gene RUNX3. Methylation-specific PCR data revealed specific unmethylation of a RUNX3 promoter in compound K-treated cells (Fig. 3A) and the quantitative pyrosequencing data showed a decrease in RUNX3 methylation (Fig. 3B). The unmethylation of RUNX3 in compound K-treated cells resulted in the re-expression of RUNX3 mRNA and protein (Fig. 3C and D). Mislocalization of nuclear RUNX3 protein to the cytoplasm is initially observed in various cancers, including colon cancer $(47,48)$. However, microscopic data showed that compound K-treated cells increased the RUNX3 protein localization in the nucleus (Fig. 3E).

Compound $K$ inhibits the expression and activity of DNMT1. Compound K treatment decreased DNMT1 mRNA and protein expression in a time-dependent manner (Fig. 4A and B). DNMT activity in the nuclear extract of compound $\mathrm{K}$-treated cells decreased in a time-dependent 
A

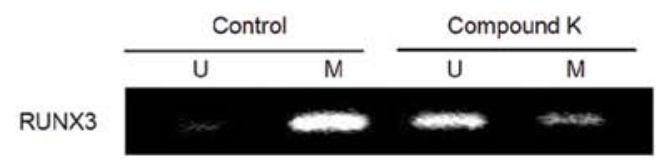

B

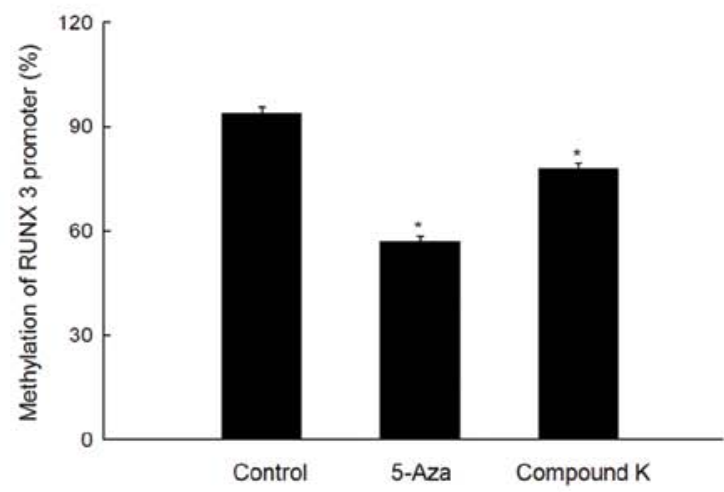

A

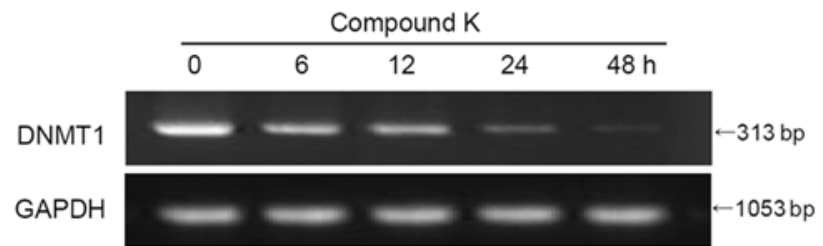

B

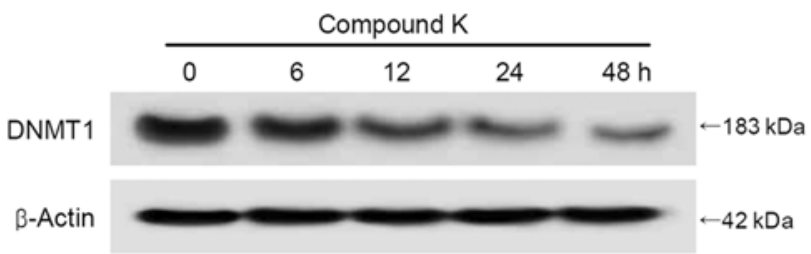
indicates the nuclear location of RUNX3 protein.

C

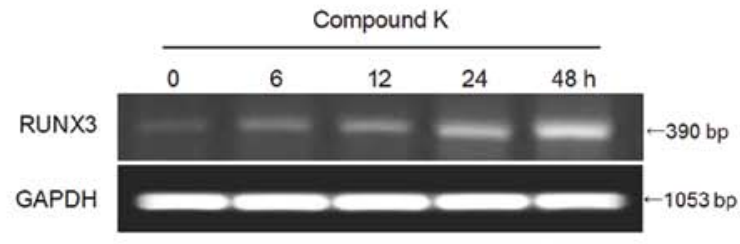

D

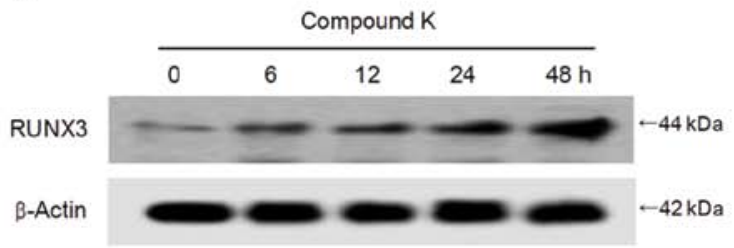

$\mathrm{E}$

DAPI

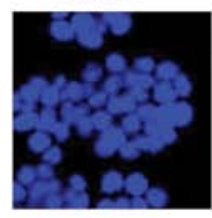

Compound $\mathrm{K}$
RUNX3
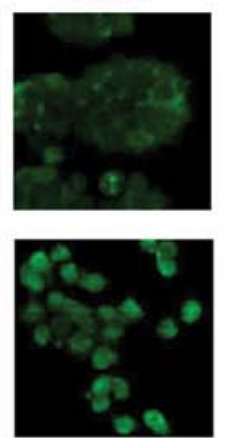

Merge
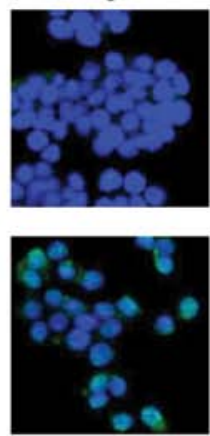

Figure 3. Effect of compound K on RUNX3 promoter methylation. (A) MS-PCR analysis of the RUNX3 promoter in HT-29 cells, and (B) pyrosequencing analysis were assessed. * $\mathrm{p}<0.05$, significantly different from the control. (C) RT-PCR and (D) western blot analysis for detecting RUNX3 mRNA and protein expression, respectively. (E) Confocal image showing the location of RUNX3 (green). DAPI staining indicates the location of the nucleus (blue), and the merged image

\section{C}

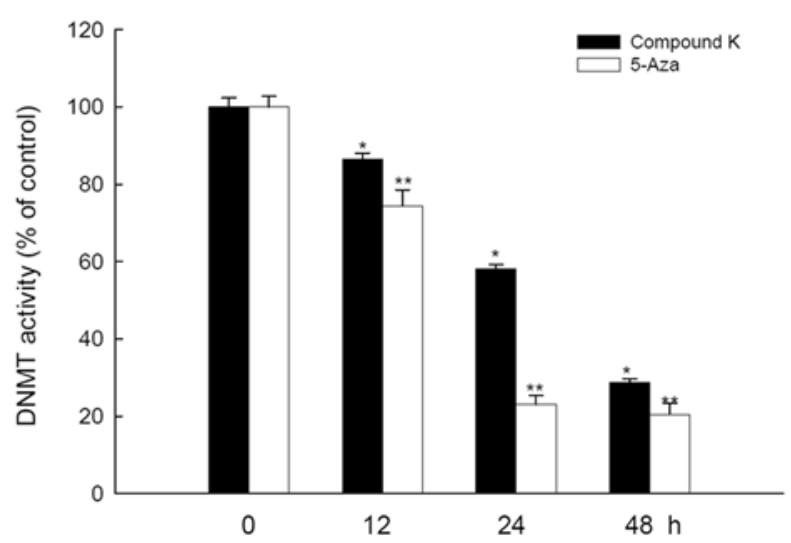

Figure 4. Compound K reduces methylation of the RUNX3 by inhibiting DNMT1. (A) RT-PCR and (B) western blot analysis for detecting DNMT1 mRNA and protein expression, respectively. (C) DNMT activity detected using a DNA methyltransferase activity assay kit. ${ }^{*}$ and ${ }^{* *}$ significantly different from the control of compound K- or 5-Aza-treated cells, respectively $(\mathrm{p}<0.05)$. 
A

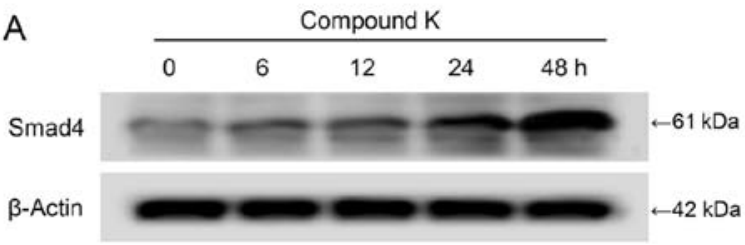

C

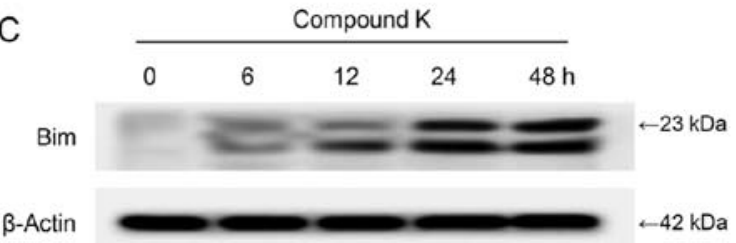

B

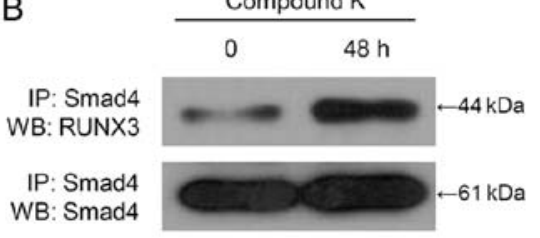

D

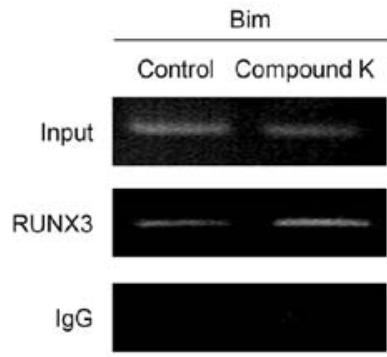

Figure 5. Compound K induces RUNX3-mediated Smad4 and Bim expression. (A) Western blot analysis showed Smad4 protein expression. (B) Interaction between RUNX3 and Smad4, examined by immune-precipitation analyses using an anti-Smad4 antibody followed by western blot analysis using RUNX3 and Smad4 antibodies. (C) Expression levels of Bim was measured by western blot analysis with the Bim antibody. (D) ChIP analysis performed with the Runx3 antibody and primers for amplifying the Bim promoter region; the band shows the level of RUNX3 in the Bim promoter region. Input represents amplification of total DNA from whole cell lysates.

A

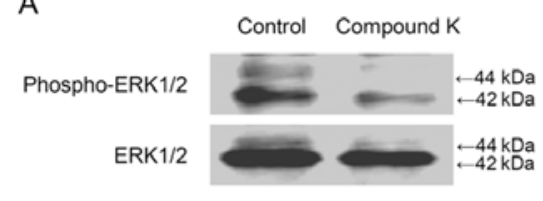

B

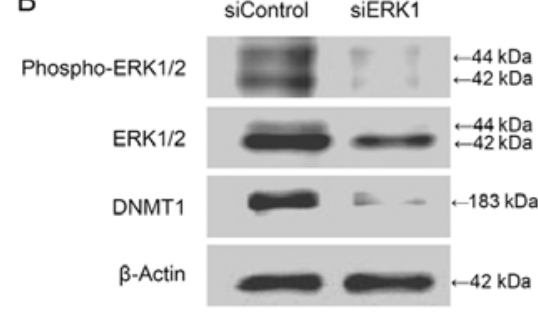

C

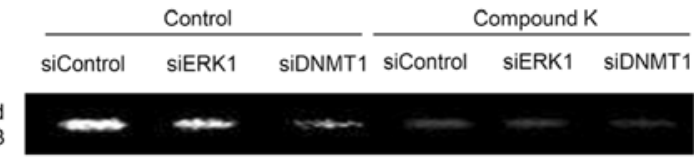

Unmethylated

RUNX3

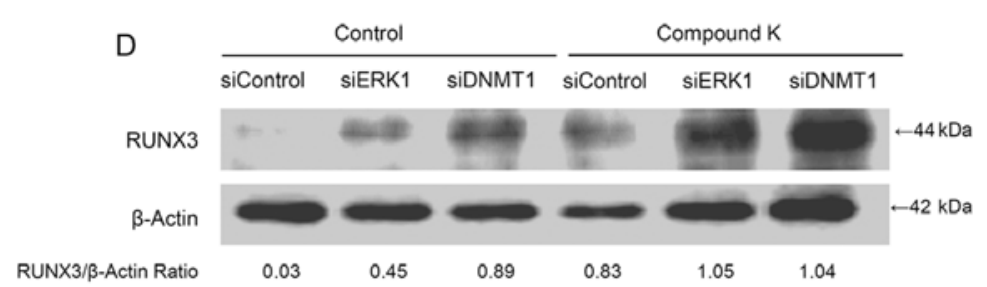

E

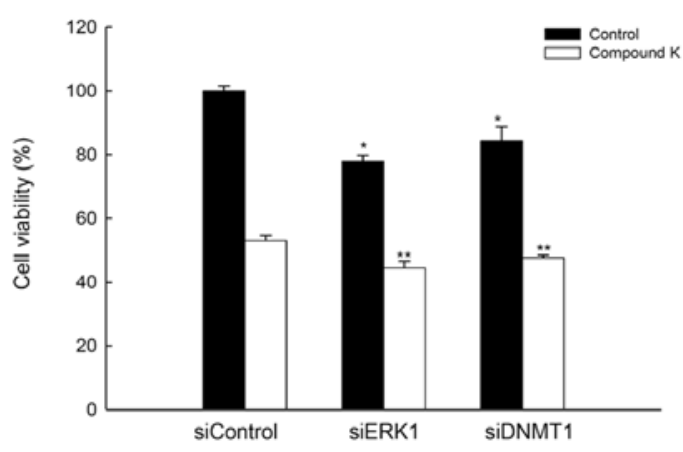

Figure 6. Compound K suppresses DNMT1 activity via the ERK pathway. (A) Western blot analysis showed phospho-ERK and ERK protein expression. (B) Western blot analysis showed phospho-ERK1/2, ERK1/2 and DNMT1 protein expression after cells were transiently transfected with ERK1- or DNMT1-specific siRNA. (C) MS-PCR performed using primers specific for the RUNX3 gene after treatment with bisulfite. (D) Western blot analysis showed RUNX3 protein expression.

(E) Cell viability assessed using the MTT test. " and ${ }^{* * *}$ significantly different from untreated-control cells or compound K-treated cells, respectively (p<0.05).

manner, similar to the mRNA and protein pattern of DNMT1 by 5-Aza (Fig. 4C).

Compound K induces RUNX3-mediated expression of Smad4 and Bim. RUNX3 cooperates with Smad3/Smad4 to activate TGF- $\beta$-dependent growth inhibition and induces apoptosis by inducing p21 and Bim $(14,42)$. Compound $\mathrm{K}$ treatment enhanced Smad4 expression in a time-dependent manner (Fig. 5A). Additionally, immunoprecipitation data showed that RUNX3 interacted with Smad4 (Fig. 5B); this complex induced Bim expression via binding to the Bim promoter region (Fig. 5C and D). 
A

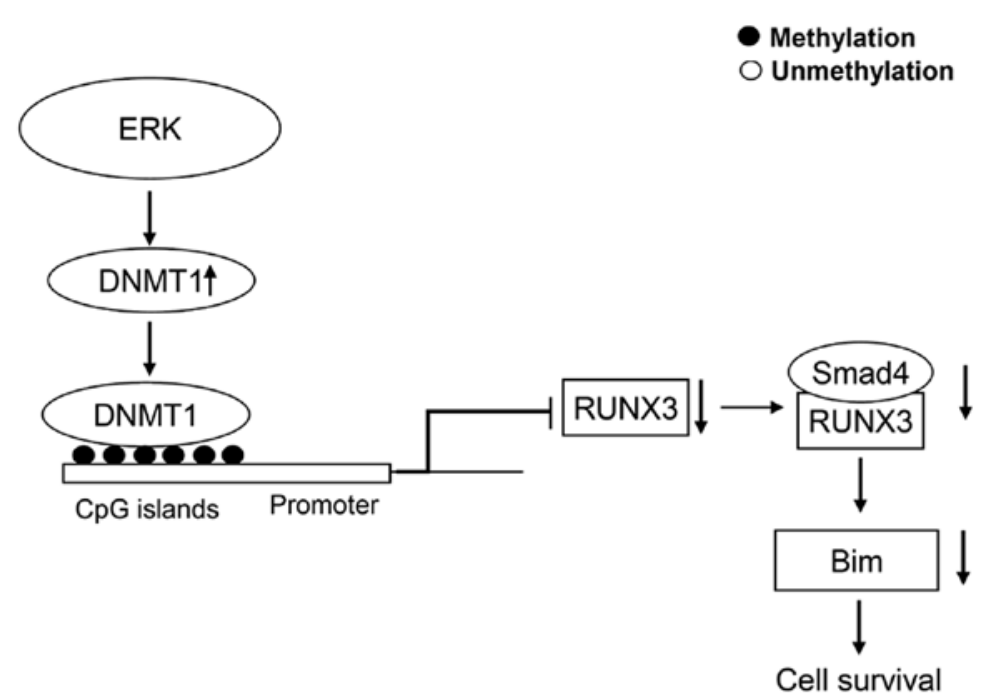

B

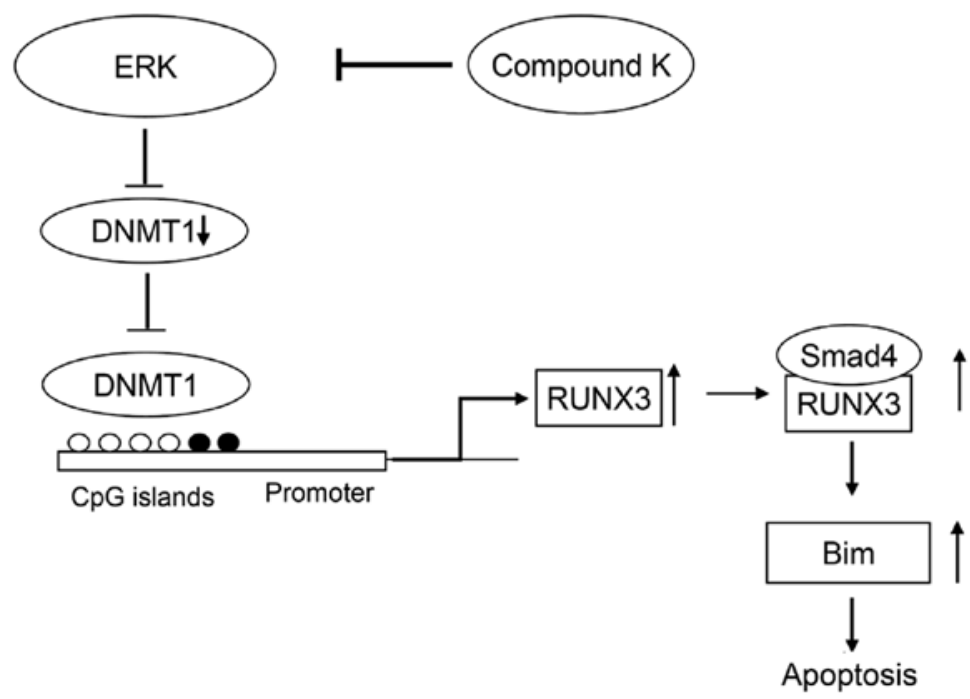

Figure 7. Proposed model of RUNX3 re-expression by compound K, wherein compound K reverses DNA methylation and RUNX3 gene silencing. (A) Model of inhibition of transcription directed by methylation of $\mathrm{CpG}$ islands in gene promoter regions. (B) Proposed model of control of gene repression by compound $\mathrm{K}$, wherein compound $\mathrm{K}$ reverses DNA methylation and gene silencing.

Compound $K$ suppresses DNMTl activity by inhibiting the ERK pathway. Lu et al (21) reported that the ERK pathway might regulate DNA methylation in colon cancer cells. In the present study, compound K-treated cells showed the decreased levels of phospho-ERK (active form) (Fig. 6A), and the decreased ERK activity by ERK1 siRNA led to reduced DNMT1 protein levels (Fig. 6B). However, siDNMT1 did not inhibit ERK expression (data not shown), indicating that ERK acts upstream of DNMT1. The siERK1 or siDNMT1 results showed that the methylation status in RUNX3 promoter decreased and the unmethylation status increased, which was enhanced by compound K (Fig. 6C). These data are consistently RUNX3 protein expression in siERK1 or siDNMT1-transfected cells with compound K treatment (Fig. 6D). The combination of compound K treatment with siERK1 or siDNMT1-transfection decreased cell viability (Fig. 6E).

\section{Discussion}

This study shows that the ginseng saponin metabolite, compound K, actively regulates cell growth in HT-29 cells. Most importantly, the results show that compound $\mathrm{K}$ can reactivate a silenced tumor suppression gene by inhibiting DNMT1 protein expression and activity. Interestingly, the effects of compound $\mathrm{K}$ were similar to those achieved using the clinically-used compound, 5-Aza $(49,50)$, a potent but toxic synthetic DNMT inhibitor. Moreover, these findings are comparable with those observed for non-toxic nutriceuticals, such as tea catechins, soy bioflavonoids, and apple polyphenols, each of which can modulate DNMT1 protein levels (51-53). Compound $\mathrm{K}$ is an active metabolite of ginsenosides that exhibits antitumor effects against various types of cancer cells, including HT-29 colorectal cancer cells $(34-40,46)$. 
DNMTs may be regulated in a cell cycle-dependent pattern in some cell types (54); for example, the level of DNMT expression is lower during the G0/G1 phase. However, cancer cells maintain higher methylation levels compared with normal cells, even during the G0/G1 phase. In our system, HT-29 cells showed methylation and silencing of RUNX3. Some reports demonstrated that lack of expression of RUNX3 in HT-29 cells can lead to inactivation of the TGF- $\beta$-mediated apoptosis pathway $(55,56)$. Re-expression of RUNX3 after compound K treatment might be the direct result of its unmethylation. Alternatively, there might be an additional transcriptional component, contributed by compound $\mathrm{K}$, once the silencing methylation is reversed. Also, our results showed compound K-treatment increased the localization of RUNX3 from cytosol into nucleus. Not only is epigenetic silencing of RUNX3 a frequent occurrence in cancer, cytoplasmic sequestration of RUNX3 was reported in a significant proportion of various cancer cases, especially, $15 \%$ of colorectal cancer $(14,18,47,48)$. These findings indicate that nuclear RUNX3 and its downstream transcriptional targets are crucial for tumor suppression. Moreover, activation of TGF- $\beta$ pathway triggered nuclear translocation of endogenous RUNX3 in SNU16 cells and this subsequently led to growth inhibition (48). With the TGF- $\beta$ pathway frequently impaired in cancer tissues, this would mean frequent cytoplasmic sequestration and, thus, functional inactivation of RUNX3. It is not known how TGF- $\beta$ elicits nuclear translocation of RUNX3. However, because SMAD and RUNX3 proteins interact (14), it is possible that perturbation of Smad nuclear import/export may promote cytoplasmic accumulation $(14,57)$. Therefore, aberrant signals from oncogenic pathways may mediate post-translational modification and subsequent cytoplasmic mislocalization of RUNX3 in cancer cells.

RUNX3 is now known to have a function in TGF- $\beta$-induced growth inhibition. After stimulation by TGF- $\beta$ cytokines, RUNX3 cooperates with SMAD proteins to directly upregulate transcription of the proapoptotic gene Bim and increase expression of the cyclin-dependent kinase inhibitor p21 in gastric cancer cells (43). Moreover, the ability of RUNX3 to target SMADs to distinct nuclear foci on stimulation by TGF- $\beta$ further strengthens the case for RUNX3 as a regulator of the TGF- $\beta$ signaling pathway (57). In this study, compound $\mathrm{K}$ induced expression of Smad4 and Bim, which is associated with RUNX3 reaction. Our results showed that compound K downregulated DNMT1 protein and mRNA levels in a timedependent manner. Therefore, it seems to that compound $\mathrm{K}$ regulated DNMT1 expression in HT-29 cells at transcriptional level. This possibility is currently being explored. The repression of DNMT1 levels by compound $\mathrm{K}$ is the likely mechanism by which silencing of these tumor suppressor genes is reversed; the downregulation of DNMT1 by siRNA is sufficient to unmethylate RUNX3 and allow re-expression. Compound K treatment also inhibited the regulation of ERK. ERK1-specific siRNA treatment downregulated DNMT1 resulting in the unmethylation of RUNX3. These results are consistent with a model in which ERK acts an upstream regulator of DNMT1 (21). In our system, suppression of DNMT1 did not inhibit ERK regulation, whereas ERK inhibition downregulated DNMT1. Downregulation of DNMT1 resulted in unmethylation of RUNX3 and expression of the RUNX3-related proteins, Smad4 and Bim, which caused cell growth inhibition.
Although it remains unknown how ERK modulates DNMT1 protein levels, we propose a model in which compound $\mathrm{K}$ influences the methylation status of RUNX3 (Fig. 7). In conclusion, compound $\mathrm{K}$ has potent demethylating activity, mediated via inhibition of ERK-DNMT signaling. The lack of toxicity associated with this ginseng saponin compound makes it an excellent and novel candidate DNMT1 inhibitor for the chemoprevention or treatment of CRC.

\section{Acknowledgements}

This study was supported by a grant from the National R\&D Program for Cancer Control, Ministry for Health and Welfare, Republic of Korea (1120340).

\section{References}

1. Kudo S, Lambert R, Allen JI, et al: Nonpolypoid neoplastic lesions of the colorectal mucosa. Gastrointest Endosc 68: S3-S47, 2008.

2. Smith JJ, Deane NG, Dhawan P and Beauchamp RD: Regulation of metastasis in colorectal adenocarcinoma: A collision between development and tumor biology. Surgery 144: 353-366, 2008.

3. Kinzler KW and Vogelstein B: Lessons from hereditary colorectal cancer. Cell 87: 159-170, 1996.

4. Grady WM and Carethers JM: Genomic and epigenetic instability in colorectal cancer pathogenesis. Gastroenterology 135: 1079-1099, 2008.

5. Wong JJ, Hawkins NJ and Ward RL: Colorectal cancer: a model for epigenetic tumorigenesis. Gut 56: 140-148, 2007.

6. Matos $\mathrm{E}$ and Brandani A: Review on meat consumption and cancer in South America. Mutat Res 506-507: 243-249, 2002.

7. Nkondjock A and Ghadirian P: Associated nutritional risk of breast and colon cancers: a population-based case control study in Montreal, Canada. Cancer Lett 223: 85-91, 2005.

8. Fearon ER and Vogelstein B: A genetic model for colorectal tumorigenesis. Cell 61: 759-767, 1990.

9. Grady WM: Genomic instability and colon cancer. Cancer Metastasis Rev 23: 11-27, 2004.

10. Herman JG and Baylin SB: Gene silencing in cancer in association with promoter hypermethylation. N Engl J Med 349: 2042-2054, 2003.

11. Levanon D, Brenner O, Negreanu V, et al: Spatial and temporal expression pattern of Runx3 (Aml2) and Runx1 (Aml1) indicates non-redundant functions during mouse embryogenesis. Mech Dev 109: 413-417, 2001.

12. Woolf E, Xiao C, Fainaru O, et al: Runx3 and Runx1 are required for CD8 T cell development during thymopoiesis. Proc Natl Acad Sci USA 100: 7731-7736, 2003.

13. Li QL, Kim HR, Kim WJ, et al: Transcriptional silencing of the RUNX3 gene by CpG hypermethylation is associated with lung cancer. Biochem Biophys Res Commun 314: 223-228, 2004.

14. Chuang LS and Ito Y: RUNX3 is multifunctional in carcinogenesis of multiple solid tumors. Oncogene 29: 2605-2615, 2010.

15. Ragnarsson G, Eiriksdottir G, Johannsdottir JT, Jonasson JG, Egilsson $\mathrm{V}$ and Ingvarsson S: Loss of heterozygosity at chromosome $1 \mathrm{p}$ in different solid human tumours: association with survival. Br J Cancer 79: 1468-1474, 1999.

16. Tanaka K, Yanoshita R, Konishi M, et al: Suppression of tumourigenicity in human colon carcinoma cells by introduction of normal chromosome $1 \mathrm{p} 36$ region. Oncogene 8: 2253-2258, 1993.

17. Li QL, Ito K, Sakakura C, et al: Causal relationship between the loss of RUNX3 expression and gastric cancer. Cell 109: $113-124,2002$

18. Goel A, Arnold CN, Tassone P, et al: Epigenetic inactivation of RUNX3 in microsatellite unstable sporadic colon cancers. Int J Cancer 112: 754-759, 2004.

19. Jair KW, Bachman KE, Suzuki H, et al: De novo $\mathrm{CpG}$ island methylation in human cancer cells. Cancer Res 66: 682-692, 2006.

20. Rhee I, Bachman KE, Park BH, et al: DNMT1 and DNMT3b cooperate to silence genes in human cancer cells. Nature 416: 552-556, 2002. 
21. Lu R, Wang X, Chen ZF, Sun DF, Tian XQ and Fang JY: Inhibition of the extracellular signal-regulated kinase/mitogenactivated protein kinase pathway decreases DNA methylation in colon cancer cells. J Biol Chem 282: 12249-12259, 2007.

22. Santi DV, Norment A and Garrett CE: Covalent bond formation between a DNA-cytosine methyltransferase and DNA containing 5-azacytosine. Proc Natl Acad Sci USA 81: 6993-6997, 1984

23. Gabbara S and Bhagwat AS: The mechanism of inhibition of DNA (cytosine-5-)-methyltransferases by 5-azacytosine is likely to involve methyl transfer to the inhibitor. Biochem J 307: 87-92, 1995.

24. Mack GS: Epigenetic cancer therapy makes headway. J Natl Cancer Inst 98: 1443-1444, 2006.

25. Beisler JA: Isolation, characterization, and properties of a labile hydrolysis product of the antitumor nucleoside, 5-azacytidine. J Med Chem 21: 204-208, 1978.

26. Constantinides PG, Jones PA and Gevers W: Functional striated muscle cells from non-myoblast precursors following 5-azacytidine treatment. Nature 267: 364-366, 1977.

27. Lyko F and Brown R: DNA methyltransferase inhibitors and the development of epigenetic cancer therapies. J Natl Cancer Inst 97: 1498-1506, 2005.

28. Gaudet F, Hodgson JG, Eden A, et al: Induction of tumors in mice by genomic hypomethylation. Science 300: 489-492, 2003.

29. Howard G, Eiges R, Gaudet F, Jaenisch R and Eden A: Activation and transposition of endogenous retroviral elements in hypomethylation induced tumors in mice. Oncogene 27 : 404-408, 2008.

30. Deng $\mathrm{T}$ and Zhang Y: 5-Aza-2'-deoxycytidine reactivates expression of RUNX3 by deletion of DNA methyltransferases leading to caspase independent apoptosis in colorectal cancer Lovo cells. Biomed Pharmacother 63: 492-500, 2009.

31. Hong SY, Cho JY and Seo DW: Ginsenoside Rp1 inhibits proliferation and migration of human lung cancer cells. Biomol Ther 19: 411-418, 2011

32. Park JW, Lee JC, Ann S, et al: A fermented ginseng extract, BST204, inhibits proliferation and motility of human colon cancer cells. Biomol Ther 19: 211-217, 2011.

33. Seo EY and Kim WK: Red ginseng extract reduced metastasis of colon cancer cells in vitro and in vivo. J Ginseng Res 35: 315-324, 2011

34. Akao T, Kanaoka $M$ and Kobashi K: Appearance of compound $\mathrm{K}$, a major metabolite of ginsenoside Rb1 by intestinal bacteria, in rat plasma after oral administrationmeasurement of compound $\mathrm{K}$ by enzyme immunoassay. Biol Pharm Bull 21: 245-249, 1998.

35. Hasegawa H, Sung JH and Huh JH: Ginseng intestinal bacterial metabolite IH901 as a new anti-metastatic agent. Arch Pharm Res 20: 539-544, 1997.

36. Quan LH, Cheng LQ, Kim HB, et al: Bioconversion of ginsenoside Rd into compound K by Lactobacillus pentosus DC101 isolated from Kimchi. J Gins Res 34: 288-295, 2010

37. Kang KA, Kim YW, Kim SU, et al: G1 phase arrest of the cell cycle by a ginseng metabolite, compound K, in U937 human monocytic leukamia cells. Arch Pharm Res 28: 685-690, 2005.

38. Kang KA, Lim HK, Kim SU, et al: Induction of apoptosis by ginseng saponin metabolite in U937 human monocytic leukemia cells. J Food Biochem 29: 27-40, 2005.

39. Chae S, Kang KA, Chang WY, et al: Effect of compound K, a metabolite of ginseng saponin, combined with gamma-ray radiation in human lung cancer cells in vitro and in vivo. J Agric Food Chem 57: 5777-5782, 2009.
40. Kim AD, Kang KA, Zhang R, et al: Ginseng saponin metabolite induces apoptosis in MCF-7 breast cancer cells through the modulation of AMP-activated protein kinase. Environ Toxicol Pharmacol 30: 134-140, 2010

41. Ito K, Lim AC, Salto-Tellez M, et al: RUNX3 attenuates betacatenin/T cell factors in intestinal tumorigenesis. Cancer Cell 14: 226-237, 2008

42. Ku JL, Kang SB, Shin YK, et al: Promoter hypermethylation downregulates RUNX3 gene expression in colorectal cancer cell lines. Oncogene 23: 6736-6742, 2004.

43. Yano T, Ito K, Fukamachi $\mathrm{H}$, et al: The RUNX3 tumor suppressor upregulates Bim in gastric epithelial cells undergoing transforming growth factor beta-induced apoptosis. Mol Cell Biol 26: 4474-4488, 2006.

44. Tong DD, Jiang Y, Li M, et al: RUNX3 inhibits cell proliferation and induces apoptosis by TGF-beta-dependent and -independent mechanisms in human colon carcinoma cells. Pathobiology 76: 163-169, 2009.

45. Kodach LL, Jacobs RJ, Heijmans J, et al: The role of EZH2 and DNA methylation in the silencing of the tumour suppressor RUNX3 in colorectal cancer. Carcinogenesis 31: 1567-1575, 2010.

46. Lee IK, Kang KA, Lim CM, et al: Compound K, a metabolite of ginseng saponin, induces mitochondria-dependent and caspase-dependent apoptosis via the generation of reactive oxygen species in human colon cancer cells. Int J Mol Sci 11: 4916-4931, 2010

47. Subramaniam MM, Chan JY, Soong R, et al: RUNX3 inactivation by frequent promoter hypermethylation and protein mislocalization constitute an early event in breast cancer progression. Breast Cancer Res Treat 113: 113-121, 2009.

48. Ito K, Liu Q, Salto-Tellez M, et al: RUNX3, a novel tumor suppressor, is frequently inactivated in gastric cancer by protein mislocalization. Cancer Res 65: 7743-7750, 2005.

49. Mani S and Herceg Z: DNA demethylating agents and epigenetic therapy of cancer. Adv Genet 70: 327-240, 2010.

50. Howell PM, Liu Z and Khong HT: Demethylating agents in the treatment of cancer. Pharmaceuticals 3: 2022-2044, 2010.

51. Fang MZ, Wang Y, Ai N, et al: Tea polyphenol (-)-epigallocatechin-3-gallate inhibits DNA methyltransferase and reactivates methylation-silenced genes in cancer cell lines. Cancer Res 63: 7563-7570, 2003.

52. Fang MZ, Chen D, Sun Y, Jin Z, Christman JK and Yang CS: Reversal of hypermethylation and reactivation of p16INK4a, RARbeta, and MGMT genes by genistein and other isoflavones from soy. Clin Cancer Res 11: 7033-7041, 2005.

53. Fini L, Selgrad M, Fogliano V, et al: Annurca apple polyphenols have potent demethylating activity and can reactivate silenced tumor suppressor genes in colorectal cancer cells. J Nutr 137: 2622-2628, 2007.

54. Kishikawa S, Murata T, Ugai H, Yamazaki T and Yokoyama KK: Control elements of Dnmt 1 gene are regulated in cell-cycle dependent manner. Nucleic Acids Res (Suppl) 3: 307-308, 2003.

55. Torquati A, O'rear L, Longobardi L, et al: RUNX3 inhibits cell proliferation and induces apoptosis by reinstating transforming growth factor beta responsiveness in esophageal adenocarcinoma cells. Surgery 136: 310-316, 2004.

56. Yamamura $\mathrm{Y}$, Lee WL, Inoue $\mathrm{K}$, Ida $\mathrm{H}$ and Ito $\mathrm{Y}$ : RUNX3 cooperates with FoxO3a to induce apoptosis in gastric cancer cells. J Biol Chem 281: 5267-5276, 2006.

57. Zaidi SK, Sullivan AJ, van Wijnen AJ, Stein JL, Stein GS and Lian JB: Integration of Runx and Smad regulatory signals at transcriptionally active subnuclear sites. Proc Natl Acad Sci USA 99: 8048-8053, 2002. 